\title{
Control of Flow Separation Using Adaptive Airfoils
}

\author{
M.S. Chandrasekhara ${ }^{1}$ \\ Navy-NASA Joint Institute of Aeronautics \\ Department of Aeronautics and Astronautics \\ Naval Postgraduate School, Monterey, CA 93943 \\ M.C. Wilder ${ }^{2}$ \\ Navy-NASA Joint Institute of Aeronautics and \\ MCAT Institute, San Jose, CA \\ and \\ L.W. Carr ${ }^{3}$ \\ Aeroflightdynamics Directorate, Aviation Research, Development and \\ Engineering Center, U.S.Army ATCOM and, \\ Fluid Mechanics Laboratory Branch \\ NASA Ames Research Center, Moffett Field, CA 94035-1000

\begin{abstract}
Submitted to the AIAA $35^{\text {th }}$ Aerospace Sciences Meeting

January 6-9, 1997, Reno, NV
\end{abstract}

\section{Summary}

A novel way of controlling flow separation is reported. The approach involves using an adaptive airfoil geometry that changes its leading edge shape to adjust to the instantaneous flow at high angles of attack such that the flow over it remains attached. In particular, a baseline NACA 0012 airfoil, whose leading edge curvature could be changed dynamically by $400 \%$ was tested under quasi-steady compressible flow conditions. A mechanical drive system was used to produce a rounded leading edge to reduce the strong local flow acceleration around its nose and thus reduce the strong adverse pressure gradient that follows such a rapid acceleration. Tests in steady flow showed that at $M=0.3$, the flow separated at about $14 \mathrm{deg}$. angle of attack for the NACA 0012 profile but could be kept attached up to an angle of about $18 \mathrm{deg}$ by changing the nose curvature. No significant hysteresis effects

\footnotetext{
1 Associate Director and Research Professor, Assoc. Fellow AIAA, Mailing Address: M.S. 260-1, NASA Ames Research Center, Moffett Field, CA 94095

2 Research Scientist

3 Research Scientist and Group Leader, Unsteady Viscous Flows, Member AIAA
} 
were observed; the flow could be made to reattach from its separated state at high angles by changing the leading edge curvature. Interestingly, the flow over a nearly semicircular nosed airfoil was separated even at low angles. By finding a "window" of angles of attack and airfoil profiles, it appeared possible to keep the flow attached through a maneuver. The shape change also modified the multiple shocks that form over the NACA 0012 airfoil at $M=0.45$. Tests are underway to document the dynamic stall flow control that can be effected with the change of airfoil geometry using high-speed, real-time, phase-locked, interferometry. Detailed results from these tests are expected to be available for presentation at the meeting.

\section{Introduction}

It is well known that performance of aircraft and their components is severely restricted by the occurrence of flow separation. It is necessary to control and manage flow separation over airfoils and wings when sustained high lift conditions are expected. The problem is even more important in unsteady flows such as encountered over the retreating blade of a helicopter rotor, a pitching aircraft wing or a wing subjected to gust loads. This task becomes more complicated when these flows are to be controlled in the compressible regime. Ongoing research ${ }^{1,2}$ on compressibility effects on dynamic stall has shown that stall onset arises during the bursting of a separation bubble (from the strong adverse pressure gradient that develops downstream of the suction peak) or at higher Mach numbers, from separation induced by shocks that form over the airfoil. Extremely rapid flow acceleration is another attribute of the dynamic stall flow, which leads to onset of compressibility effects at freestream Mach numbers as low as 0.2 causing premature onset of stall. Thus, it is necessary to reduce the leading edge flow acceleration and the subsequent development of the adverse pressure gradient if effective flow control has to be achieved. The published literature ${ }^{3,4}$ on separation control describes many novel ideas such as blowing or suction, use of slats and slots etc. However, their effectiveness in compressible flows is not yet well established. This prompted the development of the present approach wherein the airfoil leading edge is deformed to adapt to the instantaneous flow conditions in such a way that 
the flow over it remains attached throughout the range of angles of attack of interest. As a first test in proving the concept and use of a deforming airfoil for flow control, steady flow separation control was attempted. These studies showed that many different flow regimes can result as a consequence. This paper describes some of the first results of this effort.

\section{Description of the Experiment}

\subsection{Dynamically Deforming Airfoil Leading Edge Design}

The dynamically deforming leading edge (DDLE) airfoil is a 6 in. chord section made in two parts. The first $25 \%$ is made from a custom cast, carbon-fiber composite material, built up in several laminations. Its thickness increases progressively from the leading edge where it is about 0.0002 in. thick. The rear $75 \%$ of the airfoil is machined from solid metal, and the leading edge is attached to the rear section by fasteners. The carbon-fiber skin is attached to a mandrel shaped to a NACA 0012 profile by a tang cast along with the leading edge. The mandrel, housed inside the airfoil leading edge section is connected by pins at the airfoil quarter-chord-point to two a.c. brushless servomotors that are computer driven to push or pull the airfoil leading edge and produce the required shape change. The whole mechanism has been designed for controlling two-dimensional compressible dynamic stall of an oscillating airfoil at $\mathrm{M}=0.45$, and the motors can exert the necessary axial force (torque) to drive the mandrel at rates of up to $20 \mathrm{~Hz}$ to deform the airfoil from its fully extended NACA 0012 shape to a rounded nose in less than 20 millisec. and return to the NACA 0012 shape within one pitch oscillation cycle of the airfoil. Encoders mounted on the motors provide exact position information of the mandrel and thus the leading edge. The entire assembly is mounted between two circular windows with L-shaped optical glass inserts for tests in the compressible dynamic stall facility at NASA Ames Research Center (CDSF) as is schematically shown in Fig. 1. The details of the facility can be found in Ref. 5. Fig. 2 shows the range of shape changes and leading curvature that can be obtained from this design. Bench tests were conducted to ensure that the deformation is two-dimensional all along the span of the DDLE. This is very important especially since 
other methods of producing a DDLE yielded strongly three-dimensional shape changes which are not acceptable for the present purpose (Ref. 6).

\subsection{Details of the Experiments}

These first experiments were carried out in steady flow to prove the concept that a separated flow could be made to reattach by dynamically deforming an airfoil leading edge. The Mach number of the experiments ranged from 0.3 to 0.45 in increments of 0.05 . The angle of attack was varied from $6 \mathrm{deg}$ to $20 \mathrm{deg}$, with emphasis over the range where the flow was initially separated. The measurement technique used was point diffraction interferometry(PDI), which has been described in Ref. 1 and 2 in detail. The control of the brushless motors provides a way to hold the airfoil leading edge at any desired position (i.e. curvature) in order to completely document the flow using PDI during a sweep over a range of angles of attack. Alternatively, the airfoil angle of attack could be set at a fixed value and the leading edge curvature varied. (The controls also permit deforming the airfoil while it is oscillating from a fixed angle of attack at specified rates. This latter option will be exercised in the dynamic stall tests that are about to begin.) PDI images were obtained for each airfoil position and angle of attack. These were analyzed to determine whether the flow was separated and also to categorize the type of stall that occurred over the airfoil in order to assess the performance of the instantaneous airfoil shape.

In what follows, the results of this preliminary analysis will be described. A full analysis and some recommendations of the desired shapes at various angles of attack where the flow is otherwise stalled will be presented at the conference. It is also expected that some dynamic stall control results will also be available which will be included at that time. 


\section{Results and Discussion}

\subsection{Qualitative Description of Flow at $\mathrm{M}=0.3$}

Fig. 3 presents four interferogram images at $\mathrm{M}=0.3$ for an angle of attack of $18 \mathrm{deg}$. The first image is for the NACA 0012 shape (fully extended leading edge of the DDLE model). Earlier studies ${ }^{7}$ have shown that the NACA 0012 airfoil experiences leading edge stall at $14 \mathrm{deg}$; the flow has remained separated at $18 \mathrm{deg}$ as well. The second image in the figure - obtained when the leading edge has been retracted by 0.0128 in. - shows the flow to be fully attached (since the density contours are following the airfoil nose and turn smoothly parallel to the surface, like streamlines in attached flows). These contours indicate that the flow has reattached over the airfoil. This is also evidenced by the large number of fringes near the leading edge which points to the re-production of a large suction peak once the flow reattaches. In the third image, corresponding to a leading edge position of $0.0256 \mathrm{in}$. from the baseline leading edge of the fringes over the airfoil have flattened out with those on the downstream side lifting-off the surface. But, since the suction fringes still follow the "normal" pattern, the flow at this condition is experiencing a mixed-stall behavior with possible trailing edge separation creeping towards the leading edge and initiating stall. The last frame in this figure clearly shows leading edge stall again as the shear layer has separated from the airfoil nose. The airfoil radius of curvature at this condition is large and it is nearly semi-circular. These images demonstrate the range of flow regimes possible at this high angle of attack. Similar pictures were obtained at other angles of attack and Mach numbers.

\subsection{Airfoil Performance at Different Leading Edge Curvatures}

The flow over the airfoil at different angles and leading edge shapes is summarized in Fig. 4 as a plot of the various flow regimes that appeared in the experiments at $M=0.3$. It is clear that the flow is attached at low angles for all shapes (in the shaded area). Static stall occurred at $14 \mathrm{deg}$ for the NACA 0012 profile, beyond which trailing edge stall first started accompanied by a gradual loss of the suction peak (as measured by a counting of 
the fringes), eventually leading to leading edge stall. No significant changes in the flow were noticed until the nose was pulled back a distance of about $0.0256 \mathrm{in}$. (The concomitant change in the radius of curvature was large, about $100 \%$ higher than the NACA 0012 nose radius); at this position the flow became reattached and remained so at angles of attack up to $19 \mathrm{deg}$. A noticeable effect as the curvature was decreased was the sudden decrease in the tunnel noise once the flow became attached. With further decrease, a bubble formed around the 6-8\% chord location, however, the external flow remained attached. Hence, the overall flow was classified as attached. As the leading edge was retracted further, the flow abruptly stalled from the leading edge. Interestingly, as a semi-circular nose shape was approached, the flow began to experience leading edge stall at much lower angles of attack when compared to the NACA 0012 profile. Thus, it appears that a "window" of airfoil shapes is present in which the flow remains attached or can be made to reattach if it has already separated. A similar flow regime map has been drawn for $\mathrm{M}=0.35$ in Fig. 5. The major difference that is seen is a decrease in angles of attack when the above mentioned regimes occur and also a much narrower window where quasi-steady flow control can be successfully attained. This window provides a new and extended flight envelope for a wing to maneuver by constantly pursuing the "right" airfoil shape if its angle of attack is increased beyond the static stall angle.

Fig. 6 shows the flow patterns observed for $M=0.45$ in steady flow. The baseline profile stalled at about $11 \mathrm{deg}$. However, as the nose radius of curvature was increased, the flow could be made to stay attached to about $12-13 \mathrm{deg}$. But, as the nose became more rounded, the flow separated from a location of about $8-10 \%$ chord length from the leading edge. Interestingly, the airfoil continued to develop greater suction levels with increase in angle of attack. However, as the trailing edge stall propagated towards the leading edge, fully developed leading edge stall occurred. Another noteworthy point here is that there were a series of shocks that were present for most of the attached cases. But, as the airfoil became more rounded, the shocks appeared weaker than those found over the NACA 0012 profile, since their location moved downstream to points where the minimum $C_{p}$ was smaller at the root of the shocks, despite the fact that the airfoil itself developed higher suction peaks. 


\subsection{Development of Suction Peak for Different Shapes}

Fig. 7 presents the development of peak suction at $M=0.3$ for some of the different leading edge radii that were created during the tests. The numbers indicated on the graph correspond to the progressively increasing nose radius when compared to the NACA 0012 profile. It is clear that all profiles with the exception of shape 12 behave in the manner expected with the peak suction increasing with increasing angle of attack. It also shows that the NACA 0012 profile developed slightly higher suction levels. However, when this figure is interpreted in conjunction with Fig. 4 for $\mathrm{M}=0.3$, it seems that by carefully

shaping the airfoil from the baseline profile to shape 10 for angles of attack from $14-18$ $\mathrm{deg}$, the airfoil can be steadily made to produce lift with no increase in drag since the flow is attached and thus, the deforming airfoil concept is successfully demonstrated. From such a parametric study, an "optimal" shape history can be generated and used as the different flight conditions warrant.

A more detailed analysis of the large number of interferograms that were obtained during the study is ongoing. These results and some unsteady flow results which are expected to be available soon will be included in the full length paper.

\section{Conclusions}

A novel concept for separation flow control using dynamically deforming airfoils has been demonstrated in steady compressible flows. The results presently available show that attached flow can be maintained up to about $18 \mathrm{deg}$ at $\mathrm{M}=0.3$ by suitably deforming the airfoil. Parametric testing has revealed that an optimal shape history can be devised for such airfoils to sustain attached flow over a range of flow conditions. Control seems possible at $\mathrm{M}=0.45$ even when shocks that form locally influence the flow considerably.

\section{References}

${ }^{1}$ Carr, L.W., Chandrasekhara, M.S. and Brock, N., "A Quantitative Study of Unsteady Compressible Flow on an Oscillating Airfoil", Journal of Aircraft, Vol. 31, No. 4, 
Jul. - Aug. 1994, pp. $892-898$.

${ }^{2}$ Chandrasekhara, M.S., and Carr, L.W., "Compressibility Effects on Dynamic Stall of Oscillating Airfoils", AGARD-CP-552, Aug. 1995, pp. 3.1 - 3.15.

${ }^{3}$ Alrefai, M. and Acharya, M., "Controlled Leading-Edge Suction for the Management of Unsteady Separation over Pitching Airfoils", AIA A Paper 95-2188, Jun. 1995

${ }^{4}$ Carr, L.W. and McAlister, K.W., "The Effect of a Leading-Edge Slat on the Dynamic Stall of an Oscillating Airfoil", AIA A Paper 89-3599, Oct. 1983.

${ }^{5}$ Carr, L.W., and Chandrasekhara, M.S., "Design and Development of a Compressible Dynamic Stall Facility", Journal of Aircraft, Vol. 29, No. 3, pp. 314-318.

${ }^{6}$ Wilder, M.C., "Control of Unsteady Separated Flow Associated with the Dynamic Stall of Airfoils", Final Report 95-09, MCAT Institute, San Jose, CA, Jan. 1995.

${ }^{7}$ Chandrasekhara, M.S., Wilder, M.C., and Carr, L.W., "Reynolds Number Influence on 2-D Compressible Dynamic Stall", AIA A Paper 96-0073, Jan. 1996. 


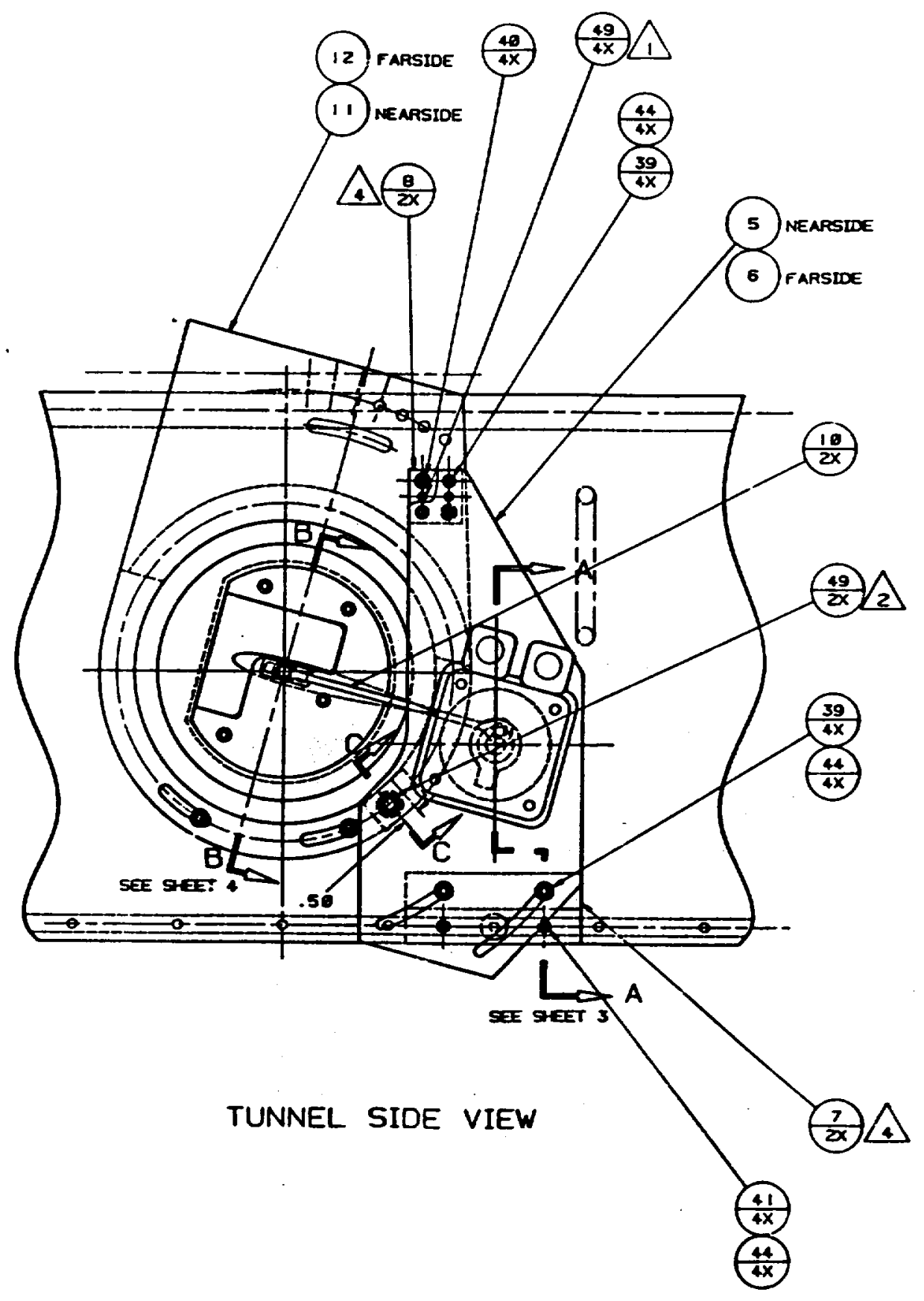

Fig. 1. Schematic of the DDLE airfoil and Actuator Mechanism. 


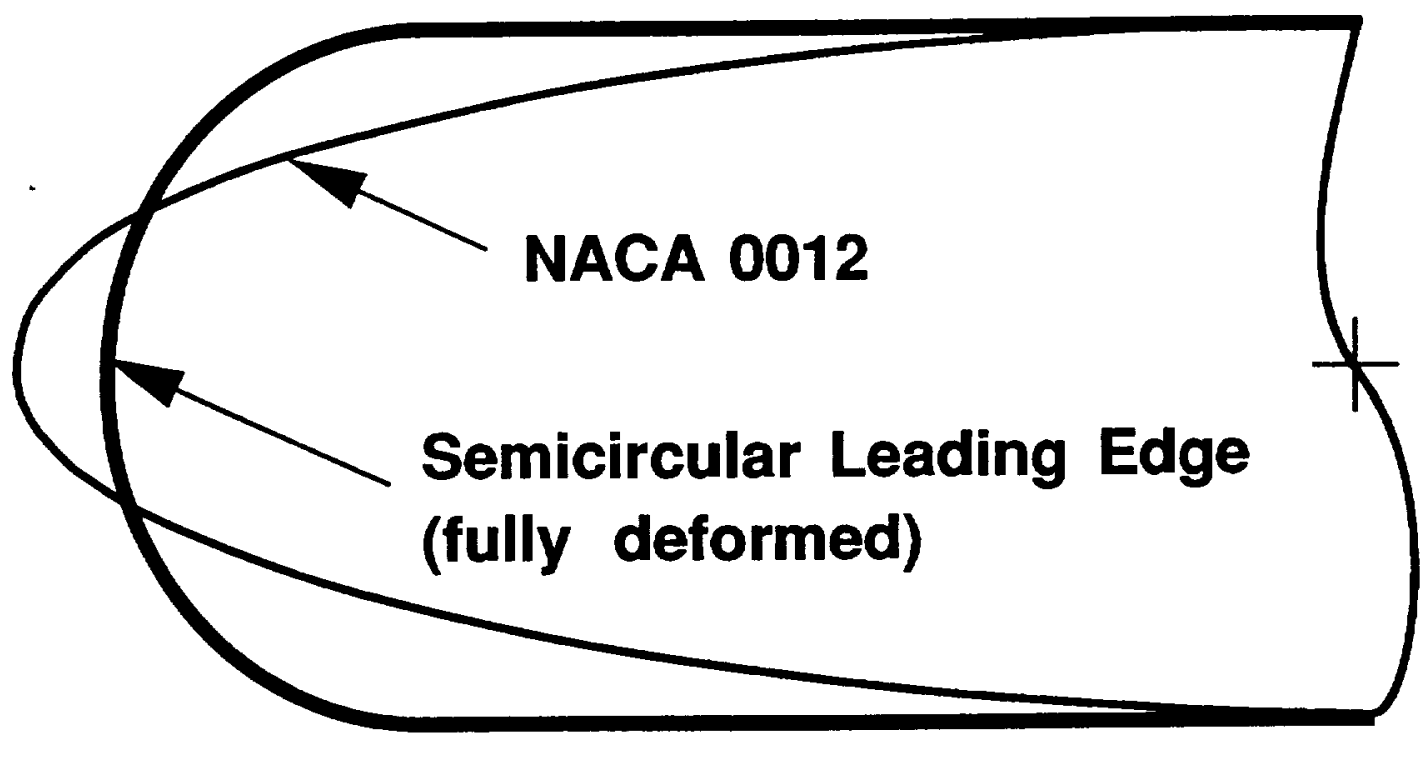

Fig. 2. Sketch Illustrating the Limiting Shapes of the DDLE Surface. 
(a)

(b)

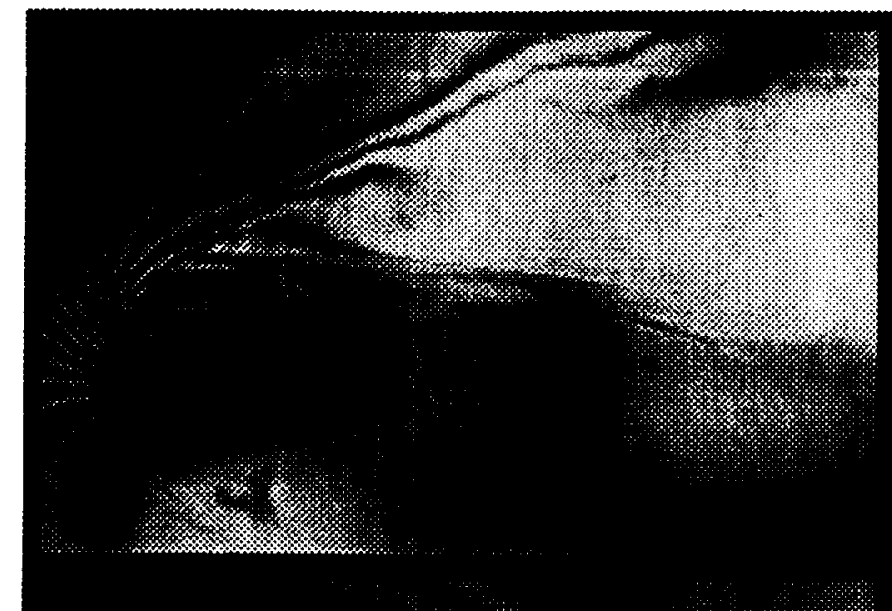

(c)

$\alpha$

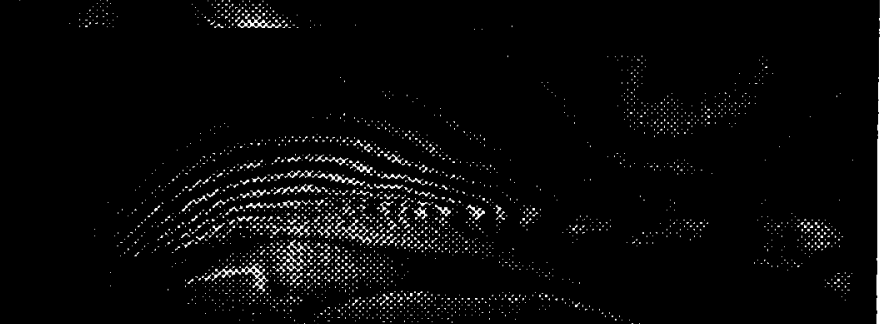

(d)

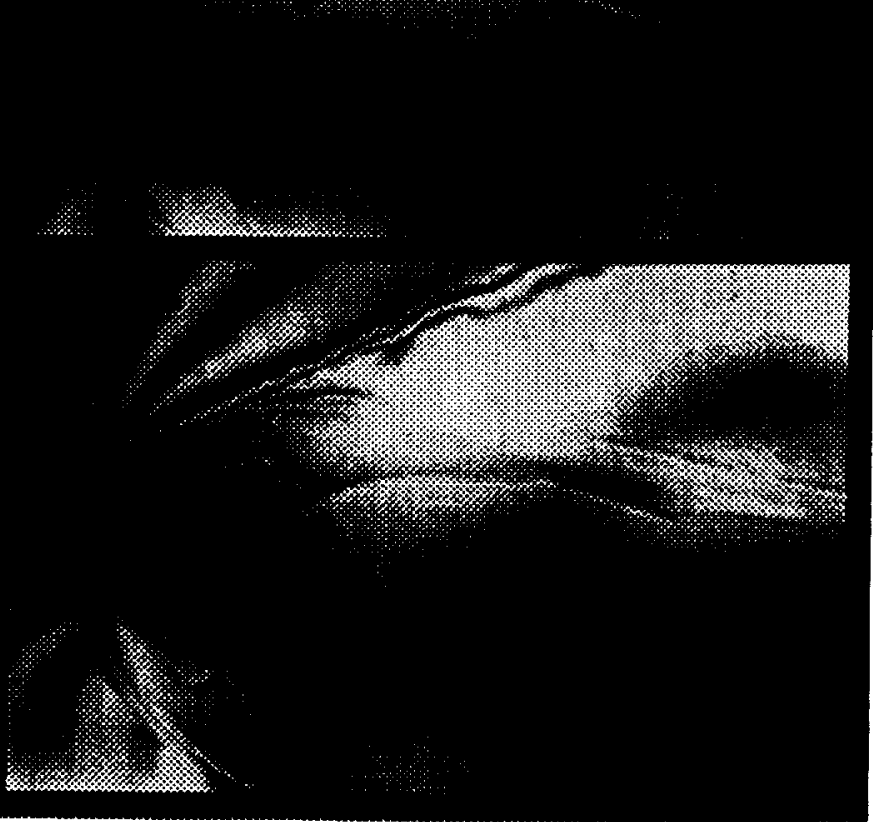

Fig. 3. Effect of Leading-Edge Shape on the Flow Field at $\alpha=18.0^{\circ}$ for $M=0.3, k=0$ :

(a) $s=0$ (leading-edge stall), (b) $s=9$ (attached flow), (c) $s=13$ (separation bubble),

(d) $s=17$ (leading-edge stall). 


\section{DDLE Flow Control \\ $M=0.3$, steady flow}

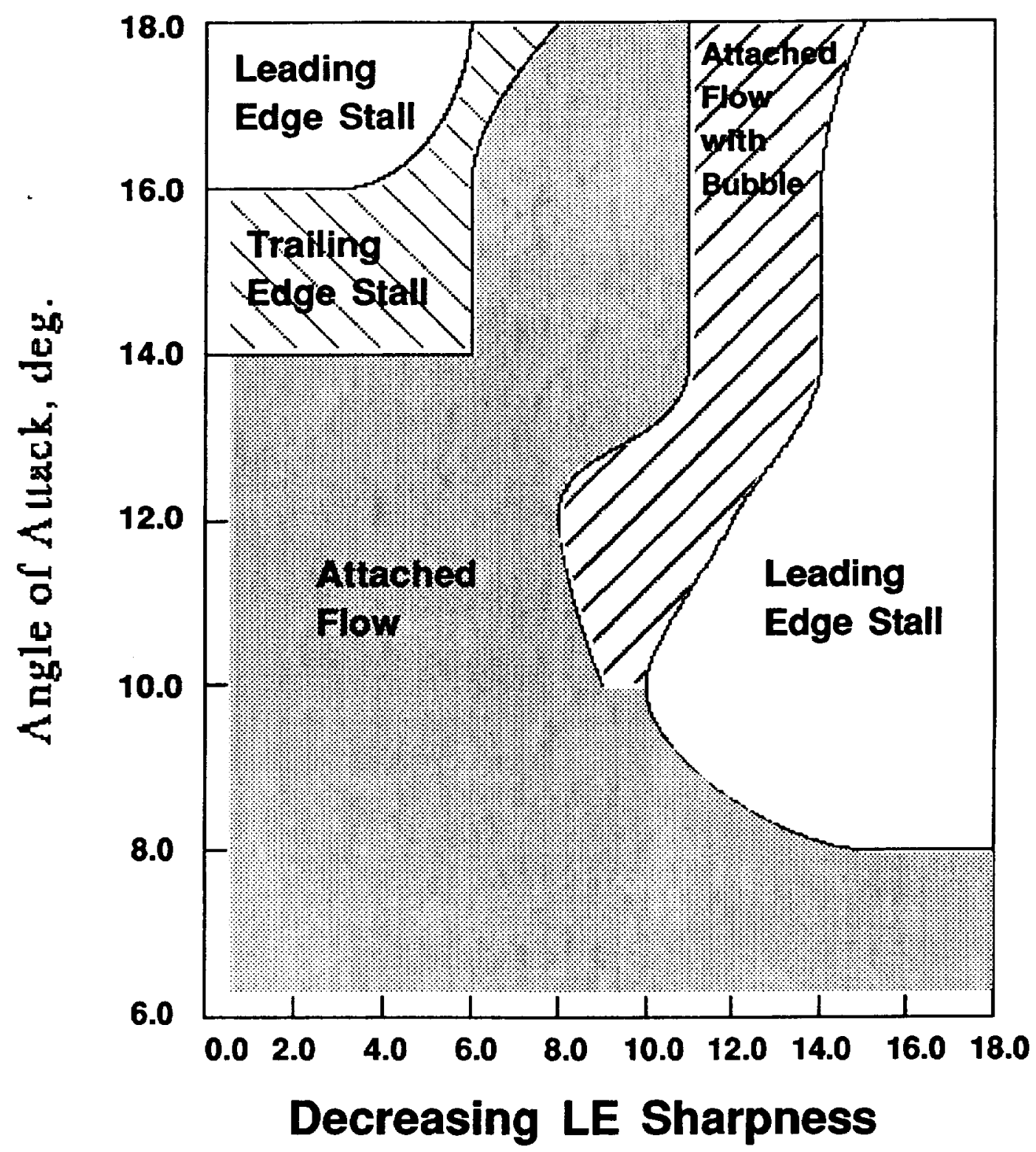

Fig. 4. Flow regimes for varying angle of attack and leading edge shape: $\mathbf{M}=0.3$. 


\section{DDLE Flow Control \\ $\mathrm{M}=0.35$, steady flow}

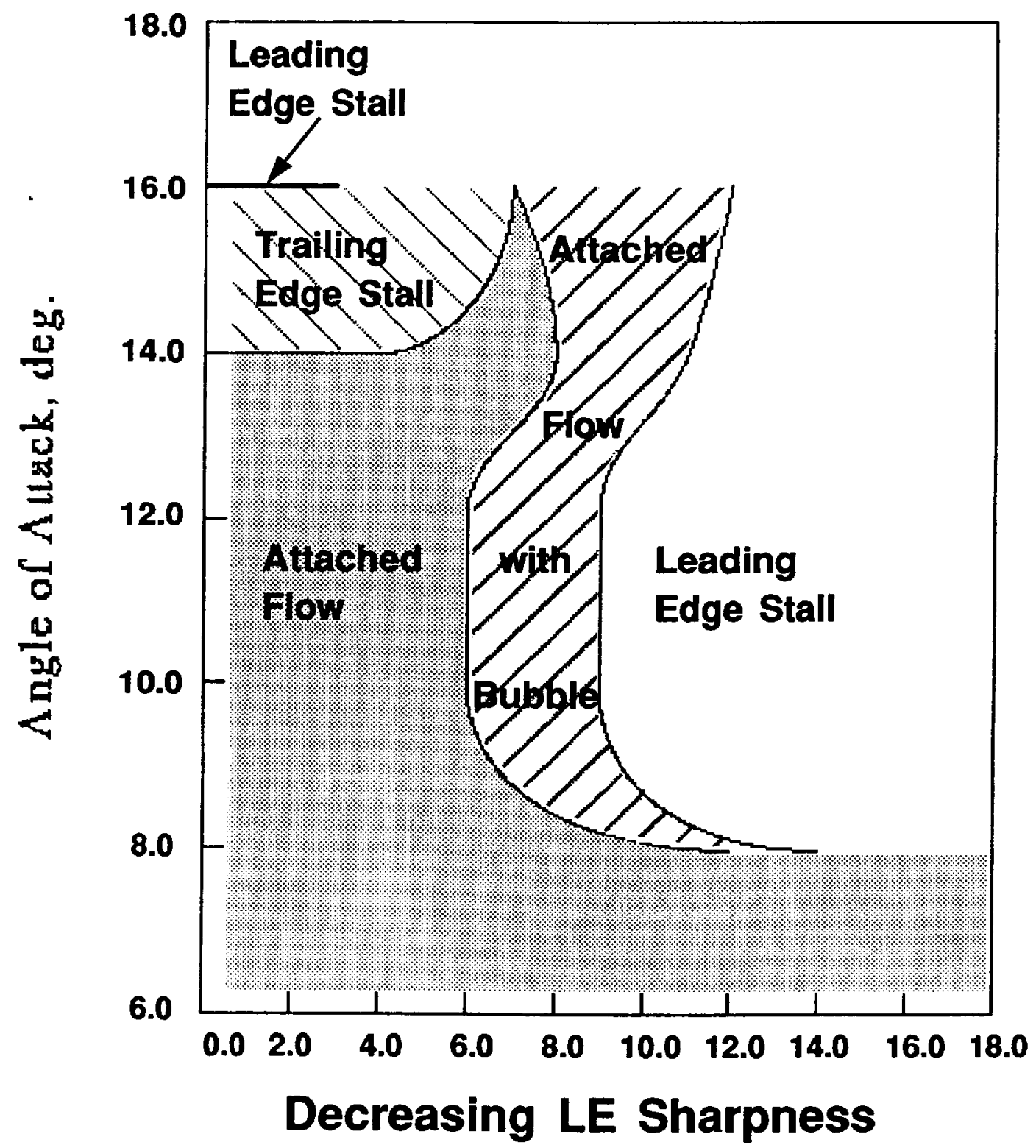

Fig. 5. Flow regimes for varying angle of attack and leading edge shape: $\mathbf{M}=0.35$. 


\section{DDLE Flow Control \\ $\mathrm{M}=0.45$, steady flow}

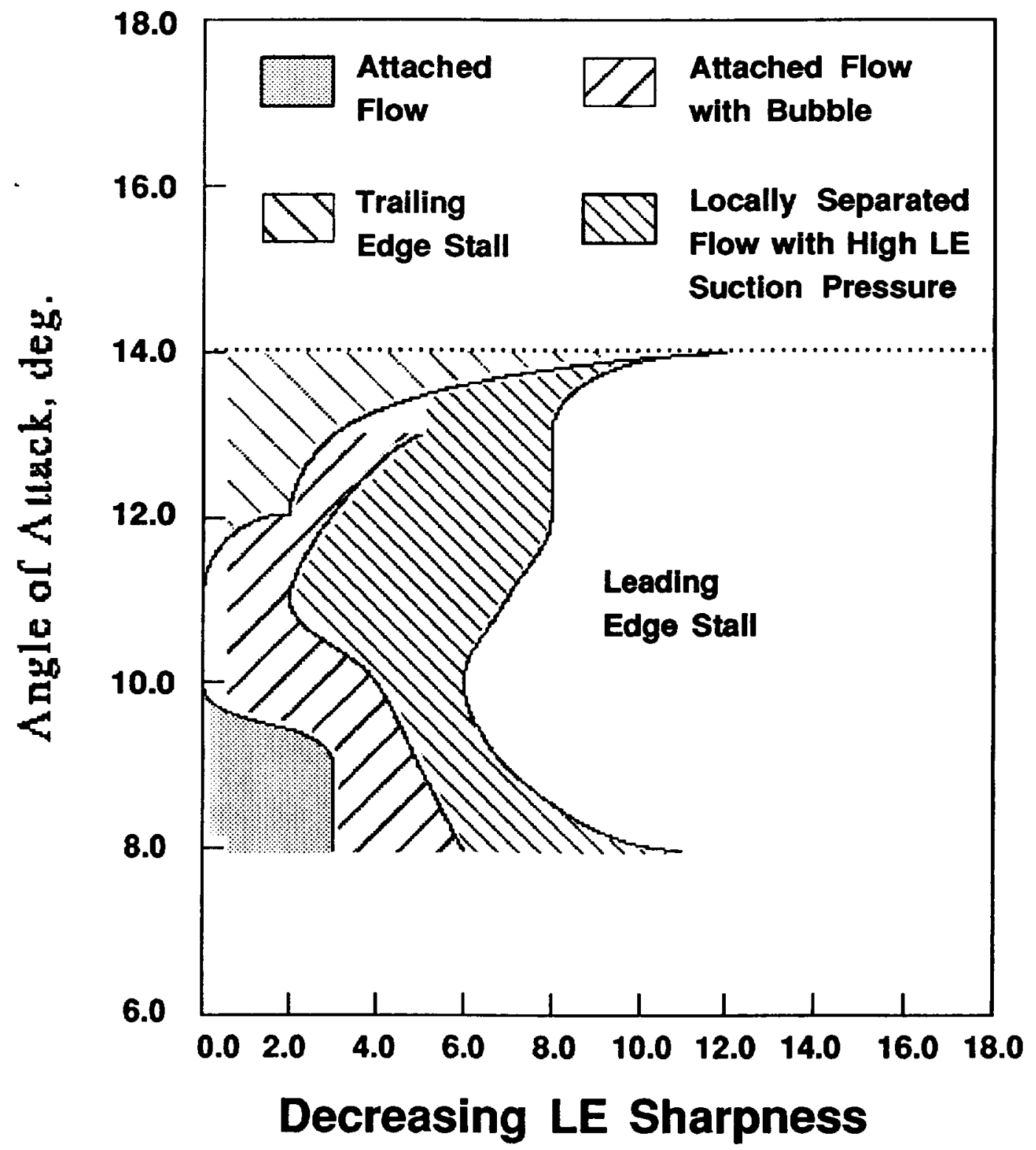

Fig. 6. Flow regimes for varying angle of attack and leading edge shape: $M=0.45$. 


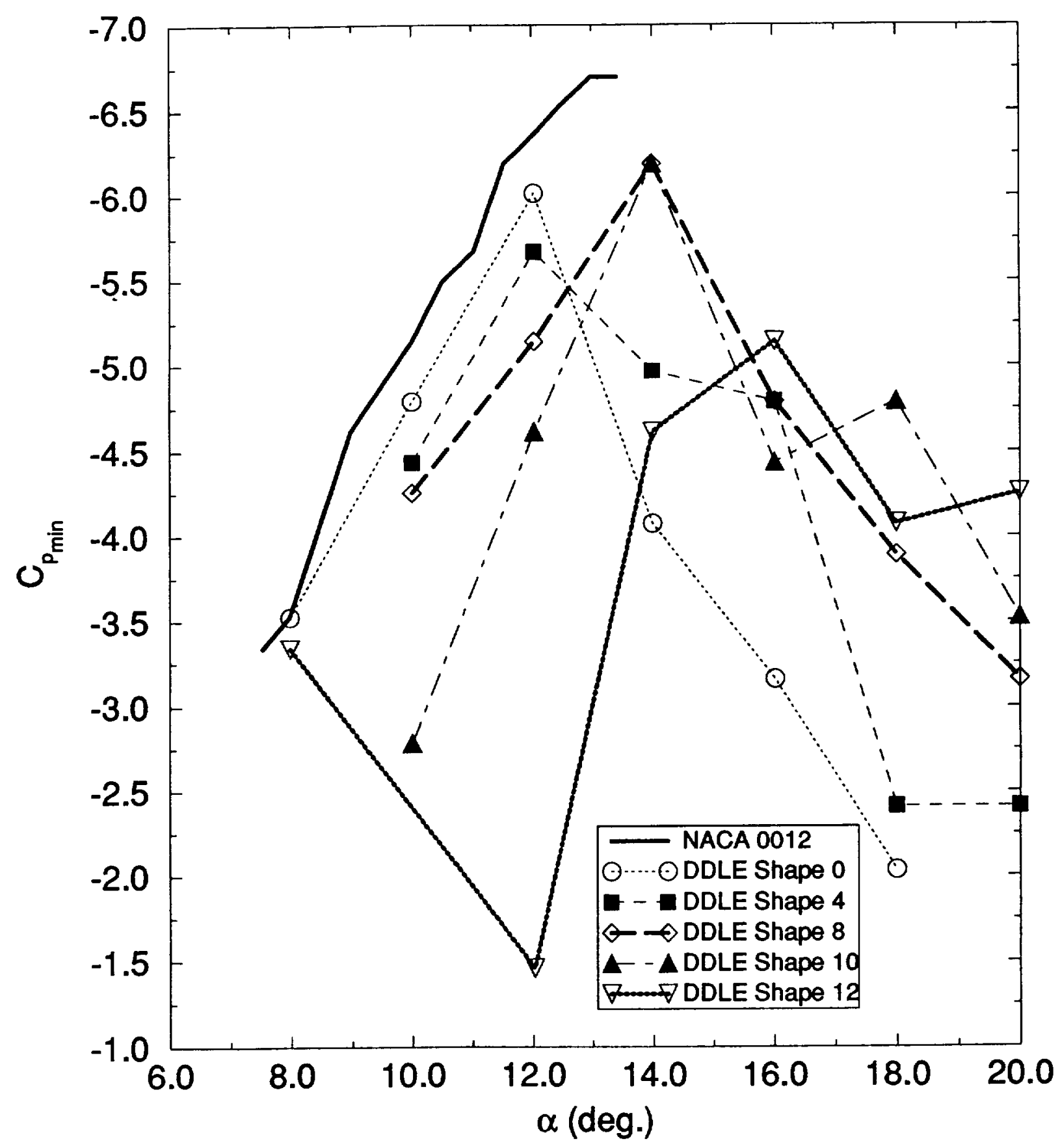

Fig. 7. Development of Peak Suction Pressure Coefficient for Different Leading-Edge Shapes: $M=0.3$. 